\title{
ON NON-PURE FORMS ON ALMOST COMPLEX MANIFOLDS
}

\author{
RICHARD HIND, COSTANTINO MEDORI, AND ADRIANO TOMASSINI
}

(Communicated by Franc Forstneric)

\begin{abstract}
In 2009 T.-J. Li and W. Zhang defined an almost complex structure $J$ on a manifold $X$ to be $\mathcal{C}^{\infty}$-pure-and-full if the second de Rham cohomology group can be decomposed as a direct sum of the subgroups whose elements are cohomology classes admitting $J$-invariant and $J$-anti-invariant representatives. It turns out (see T. Draghici, T.-J. Li and W. Zhang (2010)) that any almost complex structure on a 4-dimensional compact manifold is $\mathcal{C}^{\infty}$-pure-and-full. We study the $J$-invariant and $J$-anti-invariant cohomology subgroups on almost complex manifolds, possibly non-compact. In particular, we prove an analytic continuation result for anti-invariant forms on almost complex manifolds.
\end{abstract}

\section{INTRODUCTION}

Let $(X, J, \omega)$ be a compact Kähler manifold of complex dimension $n$. Then the celebrated Hodge decomposition theorem states that the $(p, q)$-decomposition on forms transfers at the cohomology level, namely, $H^{k}(X ; \mathbb{C}) \cong \bigoplus_{p+q=k} H_{\bar{\partial}}^{p, q}(X)$, where $H_{\bar{\partial}}^{p, q}(X)$ denotes the $(p, q)$-Dolbeault group. In order to generalize the previous decomposition to arbitrary compact almost complex manifolds, in [16] T.-J. Li and W. Zhang introduced the notion of $\mathcal{C}^{\infty}$-pure-and-full almost complex structures. An almost complex structure $J$ on a $2 n$-dimensional manifold $X$ is said to be $\mathcal{C}^{\infty}$-pure-and-full if $H^{2}(X ; \mathbb{R})=H_{J}^{(1,1)}(X)_{\mathbb{R}} \oplus H_{J}^{(2,0),(0,2)}(X)_{\mathbb{R}}$, where $H_{J}^{(1,1)}(X)_{\mathbb{R}}$ and $H_{J}^{(2,0),(0,2)}(X)_{\mathbb{R}}$ are the subgroups of the 2 -nd de Rham cohomology group of $X$, whose elements are cohomology classes represented by real pure forms of types $(1,1)$ and $(2,0)+(0,2)$, respectively, namely, the $J$ invariant, $J$-anti-invariant cohomology subgroups. More generally, $J$ is said to be $\mathcal{C}^{\infty}$-pure if $H_{J}^{(1,1)}(X)_{\mathbb{R}} \cap H_{J}^{(2,0),(0,2)}(X)_{\mathbb{R}}=\{0\}$ and $\mathcal{C}^{\infty}$-full if $H^{2}(X ; \mathbb{R})=$ $H_{J}^{(1,1)}(X)_{\mathbb{R}}+H_{J}^{(2,0),(0,2)}(X)_{\mathbb{R}}$. Similar notions of $\mathcal{C}^{\infty}$-pure and $\mathcal{C}^{\infty}$-full almost complex structures at stage $k$ can be defined. By taking the complex of $(p, q)$-currents one can define pure and full almost complex structures.

In [9], T. Drăghici, T.-J. Li and W. Zhang showed that an almost complex structure $J$ on a 4 -dimensional compact manifold $X$ is $\mathcal{C}^{\infty}$-pure-and-full, namely

Received by the editors August 30, 2011 and, in revised form, October 12, 2012 and December 14, 2012.

2010 Mathematics Subject Classification. Primary 32Q60, 53C15, 58A12.

Key words and phrases. Pure and full almost complex structure, J-invariant form, $J$-antiinvariant form.

Partially supported by Fondazione Bruno Kessler-CIRM (Trento) and by GNSAGA of INdAM. 
$H^{2}(X ; \mathbb{R})=H_{J}^{(1,1)}(X)_{\mathbb{R}} \oplus H_{J}^{(2,0),(0,2)}(X)_{\mathbb{R}}$. The last decomposition can be considered as a Hodge decomposition for 4-dimensional almost complex compact manifolds. Furthermore, by setting

$$
\mathcal{K}_{J}^{t}=\left\{[\omega] \in H^{2}(X ; \mathbb{R}) \mid \omega \text { is symplectic and } J \text { is } \omega \text {-tamed }\right\}
$$

as the tamed symplectic cone and

$$
\mathcal{K}_{J}^{c}=\left\{[\omega] \in H^{2}(X ; \mathbb{R}) \mid \omega \text { is symplectic and } J \text { is } \omega \text {-compatible }\right\}
$$

as the compatible symplectic cone, if $J$ is a complex structure on a compact manifold $M$ which admits a Kähler metric, then

$$
\mathcal{K}_{J}^{t}=\mathcal{K}_{J}^{c}+\left(\left(H_{\bar{\partial}}^{2,0}(X) \oplus H_{\bar{\partial}}^{0,2}(X)\right) \cap H^{2}(X ; \mathbb{R})\right)
$$

(see [16]). For a non-integrable almost complex structure $J$, in [16] it is proved that if $J$ is a $\mathcal{C}^{\infty}$-full almost complex structure which carries an almost Kähler metric, then

$$
\mathcal{K}_{J}^{t}=\mathcal{K}_{J}^{c}+H_{J}^{(2,0),(0,2)}(X)_{\mathbb{R}}
$$

Hence the subgroup $H_{J}^{(2,0),(0,2)}(X)_{\mathbb{R}} \subset H^{2}(M ; \mathbb{R})$ can be thought of as a generalization of the real Dolbeault group $\left(H_{\bar{\partial}}^{2,0}(X) \oplus H_{\bar{\partial}}^{0,2}(X)\right) \cap H^{2}(X ; \mathbb{R})$ to the non-integrable case.

For other results on $\mathcal{C}^{\infty}$-pure-and-full almost complex structures, see e.g. [10, [1], 3].

In dimension higher than four, there are examples of non- $\mathcal{C}^{\infty}$-pure and non- $\mathcal{C}^{\infty}$ full almost complex structures on compact manifolds. Nevertheless, in [9] and [12] it is proved that if $(X, \omega)$ is a compact symplectic manifold of dimension $2 n$, then every $\omega$-compatible almost complex structure $J$ is $\mathcal{C}^{\infty}$-pure.

In this paper we are interested in studying the $J$-invariant and $J$-anti-invariant respectively cohomology subgroups on almost complex manifolds $X^{2 n}$, possibly non-compact. In particular we will search for pairs $(\omega, J)$ where $\omega$ is either a $J$ invariant or $J$-anti-invariant closed 2 -form. We are also interested in determining whether $\omega$ can also be taken to be symplectic. We note that symplectic forms always have compatible almost complex structures, which are invariant in our language. Therefore in the symplectic case we will focus on finding anti-invariant almost complex structures.

After some preliminaries in section 1, in section 2 we start with the observation that if $n$ is odd and $(\omega, J)$ is an anti-invariant pair, then $\omega^{n}$ is identically zero; see Proposition 2.1. In particular $\omega$ cannot be symplectic. However it is not true that lower powers of $\omega$ must vanish, even in cohomology. In Example 2.2 we provide an example of a 2-cohomology class on a 6-dimensional compact nilmanifold $N$ endowed with a left-invariant almost complex structure $J$ which belongs to $H_{J}^{(1,1)}(N)_{\mathbb{R}} \cap H_{J}^{(2,0),(0,2)}(N)_{\mathbb{R}}$ and whose square is non-zero.

In searching for anti-invariant forms with $\omega$ symplectic, we must assume that $n$ is even. We consider the case of open manifolds. Theorem 3.1 says that on an open symplectic 4 manifold $\left(X^{4}, \omega\right)$ there exists an almost complex structure $J$ such that $\omega$ is $J$-anti-invariant if and only if the tangent bundle is symplectically trivial. However Proposition 3.3 shows that this is no longer true in higher dimensions. If we do not fix the symplectic form, Theorem 3.5 says that given any open manifold $X^{2 n}$ with $n$ even and $T X$ trivial, and any cohomology class $\mathfrak{a} \in H^{2}(X, \mathbb{R})$, there exists an anti-invariant pair $(\omega, J)$ with $[\omega]=\mathfrak{a}$. 
In the remainder of the paper we are motivated by the problem of finding antiinvariant pairs $(\omega, J)$ in a given cohomology class without the restrictive symplectic assumption. As in Proposition 2.1 it is not hard to show, for example, that on a 6manifold such an $\omega$ must everywhere have rank 0 or 4 . There are no other pointwise restrictions. Still, this seems restrictive, but we show in section 5 that for many codimension 2 submanifolds $W$ we can find closed forms $\omega$ supported in arbitrarily small tubular neighborhoods such that $\omega$ has everywhere rank 0 or 4 and $[\omega]$ is Poincaré dual to $W$. Given this, it is natural to ask whether anti-invariant pairs can be constructed using cut and paste methods.

Section 4 shows that this is impossible. Indeed, despite the rank condition, the compactly supported forms constructed in section 5 have no anti-invariant almost complex structures. This is a consequence of the unique continuation theorem; see Theorem 4.1. This says that given an anti-invariant pair $(\omega, J)$, if $\omega$ vanishes on an open subset, then it must vanish everywhere on a connected $X^{2 n}$.

\section{Preliminaries}

1.1. $\mathcal{C}^{\infty}$-pure-and-full almost complex structures. Let $X$ be a $2 n$-dimensional manifold (without boundary) and $J$ be a smooth almost complex structure on $X$. Let $\left(T^{1,0} X\right)^{*}$ and $\left(T^{0,1} X\right)^{*}$ be the bundle of complex 1-forms on $X$ of type $(1,0)$ and $(0,1)$, respectively. Denote by $\Lambda_{\mathbb{C}}^{k}(X)$ the bundle of $\mathcal{C}^{\infty}$ complex $k$-forms on $X$ and by the same symbol $\Lambda_{\mathbb{C}}^{k}(X)=\Gamma\left(M, \Lambda_{\mathbb{C}}^{k}(X)\right)$ the space of sections of $\Lambda_{\mathbb{C}}^{k}(X)$. Then, as usual, setting $\Lambda_{J}^{p, q}(X)=\Lambda^{p}\left(\left(T^{1,0} X\right)^{*}\right) \wedge \Lambda^{q}\left(\left(T^{0,1} X\right)^{*}\right)$, the space of complex $k$-forms decomposes as

$$
\Lambda_{\mathbb{C}}^{k}(X)=\bigoplus_{p+q=k} \Lambda_{J}^{p, q}(X)
$$

where $\Lambda_{J}^{p, q}(X)=\Gamma\left(M, \Lambda_{J}^{p, q}(X)\right)$.

According to the above decomposition, the space $\Lambda_{\mathbb{R}}^{k}(X)$ of real smooth differential $k$-forms has a type decomposition:

$$
\Lambda_{\mathbb{R}}^{k}(X)=\bigoplus_{p+q=k} \Lambda_{J}^{(p, q),(q, p)}(X)_{\mathbb{R}}
$$

where

$$
\Lambda_{J}^{(p, q),(q, p)}(X)_{\mathbb{R}}=\left\{\alpha \in \Lambda_{J}^{p, q}(X) \oplus \Lambda_{J}^{q, p}(X) \mid \alpha=\bar{\alpha}\right\}
$$

In particular, since $J$ acts as the identity on $\Lambda_{J}^{(1,1)}(X)_{\mathbb{R}}$ and as minus the identity on $\Lambda_{J}^{(2,0),(0,2)}(X)_{\mathbb{R}}$, sometimes we will set

$$
\Lambda_{J}^{+}=\Lambda_{J}^{(1,1)}(X)_{\mathbb{R}}, \quad \Lambda_{J}^{-}=\Lambda_{J}^{(2,0),(0,2)}(X)_{\mathbb{R}},
$$

and we will refer to them as the spaces of $J$-invariant and $J$-anti-invariant forms, respectively.

For a finite set $S$ of pairs of integers, let

$$
\mathcal{Z}_{J}^{S}=\bigoplus_{(p, q) \in S} \mathcal{Z}_{J}^{(p, q),(q, p)}, \quad \mathcal{B}_{J}^{S}=\bigoplus_{(p, q) \in S} \mathcal{B}_{J}^{(p, q),(q, p)},
$$

where

$$
\mathcal{Z}_{J}^{(p, q),(q, p)}=\left\{\alpha \in \Lambda_{J}^{(p, q),(q, p)}(X)_{\mathbb{R}} \mid d \alpha=0\right\}
$$

and

$$
\mathcal{B}_{J}^{(p, q),(q, p)}=\left\{\beta \in \Lambda_{J}^{(p, q),(q, p)}(X)_{\mathbb{R}} \mid \text { there exists } \gamma \text { such that } \beta=d \gamma\right\}
$$


Denoting by $\mathcal{B}$ the space of $d$-exact forms, we have that

$$
\frac{\mathcal{Z}_{J}^{S}}{\mathcal{B}_{J}^{S}}=\frac{\mathcal{Z}_{J}^{S}}{\mathcal{B} \cap \mathcal{Z}_{J}^{S}}=\frac{\mathcal{Z}_{J}^{S}}{\mathcal{B}} \text {. }
$$

Hence, there is a natural inclusion

$$
\rho_{S}: \mathcal{Z}_{J}^{S} / \mathcal{B}_{J}^{S} \rightarrow \mathcal{Z} / \mathcal{B}
$$

As in [16] we will write $\rho_{S}\left(\mathcal{Z}_{J}^{S} / \mathcal{B}_{J}^{S}\right)$ simply as $\mathcal{Z}_{J}^{S} / \mathcal{B}_{J}^{S}$ and we may define the cohomology subgroups

$$
H_{J}^{S}(X)_{\mathbb{R}}=\left\{[\alpha] \mid \alpha \in \mathcal{Z}_{J}^{S}\right\}=\frac{\mathcal{Z}_{J}^{S}}{\mathcal{B}} .
$$

In particular, according to the above definition, there is a natural inclusion

$$
H_{J}^{(1,1)}(X)_{\mathbb{R}}+H_{J}^{(2,0),(0,2)}(X)_{\mathbb{R}} \subseteq H^{2}(X, \mathbb{R}) .
$$

We recall the following (see [16, Definitions 2.2 and 2.3, Lemma 2.2])

Definition 1.1. A smooth almost complex structure $J$ on $X$ is said to be

- $\mathcal{C}^{\infty}$-pure if

$$
H_{J}^{(1,1)}(X)_{\mathbb{R}} \cap H_{J}^{(2,0),(0,2)}(X)_{\mathbb{R}}=\{0\}
$$

- $\mathcal{C}^{\infty}$-full if

$$
H^{2}(X ; \mathbb{R})=H_{J}^{(1,1)}(X)_{\mathbb{R}}+H_{J}^{(2,0),(0,2)}(X)_{\mathbb{R}} ;
$$

- $\mathcal{C}^{\infty}$-pure-and-full if

$$
H^{2}(X ; \mathbb{R})=H_{J}^{(1,1)}(X)_{\mathbb{R}} \oplus H_{J}^{(2,0),(0,2)}(X)_{\mathbb{R}} .
$$

1.2. Pure-and-full almost complex structures. Let $(X, J)$ be a compact $2 n$ dimensional almost complex manifold. Denote by $\mathcal{D}_{k}(X)$ the space of currents of dimension $k$ (or degree $(2 n-k)$ ), i.e., the topological dual of the space $\Lambda^{k}(X)$ of $r$-forms on $X$ (see e.g. [8], 7]). The exterior differential $d$ on $\Lambda^{\bullet}(X)$ induces a differential on $\mathcal{D}_{\bullet}(X)$, still denoted by $d$. Then the de Rham homology $H_{\bullet}(X ; \mathbb{R})$ is the cohomology of the differential complex $(\mathcal{D} \bullet(X), d)$ and $H_{d R}^{k}(X ; \mathbb{R}) \simeq H_{2 n-k}(X ; \mathbb{R})$. We will denote by $\mathcal{P}: H_{2 n-k}(X ; \mathbb{R}) \rightarrow H_{d R}^{k}(X ; \mathbb{R})$ this isomorphism.

As in the case of complex forms, the almost complex structure $J$ induces a bigrading on the space $\mathcal{D}_{k}^{\mathbb{C}}(X)$ of complex currents of dimension $k$. Hence, the spaces of currents $\mathcal{D}_{p, q}(X)$ of bidimension $(p, q)$ are defined.

Accordingly, let $H_{(2,0),(0,2)}^{J}(X)_{\mathbb{R}}$ (respectively, $\left.H_{(1,1)}^{J}(X)_{\mathbb{R}}\right)$ be the subspace of $H_{2}(X ; \mathbb{R})$ given by the homology classes represented by a real current of bidimension $(2,0)+(0,2)$ (respectively, $(1,1))$. Then we have the following definition due to T.-J. Li and W. Zhang.

Definition 1.2 ([16, Definition 2.5, Lemma 2.7]). An almost complex structure $J$ on $X$ is said to be:

- pure if

- full if

$$
H_{(2,0),(0,2)}^{J}(X)_{\mathbb{R}} \cap H_{(1,1)}^{J}(X)_{\mathbb{R}}=\{0\}
$$

$$
H_{(2,0),(0,2)}^{J}(X)_{\mathbb{R}}+H_{(1,1)}^{J}(X)_{\mathbb{R}}=H_{2}(X ; \mathbb{R}) ;
$$

- pure-and-full if it is both pure and full, i.e. if the following decomposition holds:

$$
H_{(2,0),(0,2)}^{J}(X)_{\mathbb{R}} \oplus H_{(1,1)}^{J}(X)_{\mathbb{R}}=H_{2}(X ; \mathbb{R}) .
$$


The notions of $\mathcal{C}^{\infty}$-pure-and-full and pure-and-full almost complex structures can be given in a similar way for $k$-forms and $k$-currents, respectively.

The relations between being $\mathcal{C}^{\infty}$-pure-and-full and being pure-and-full are summarized in the following.

Proposition 1.3 (see [16, Proposition 2.5], 11, Theorem 2.1]). The following relations between $\mathcal{C}^{\infty}$-pure-and-full and pure-and-full concepts hold:

$$
\begin{array}{ccc}
\mathcal{C}^{\infty} \text {-full at the } k \text {-th stage } & \Longrightarrow & \text { pure at the } k \text {-th stage } \\
\Downarrow & & \Downarrow \\
\text { full at the }(2 n-k) \text {-th stage } & \Longrightarrow & \mathcal{C}^{\infty} \text {-pure at the }(2 n-k) \text {-th stage. }
\end{array}
$$

In the sequel we will use the following notation:

$$
\begin{aligned}
\mathcal{Z}_{J}^{-} & =\mathcal{Z}_{J}^{(2,0),(0,2)}, \\
\mathcal{Z}_{J}^{+} & =\mathcal{Z}_{J}^{(1,1)}, \\
\mathcal{B}_{J}^{-} & =\mathcal{B}_{J}^{(2,0),(0,2)}, \\
\mathcal{B}_{J}^{+} & =\mathcal{B}_{J}^{(1,1)}, \\
H_{J}^{-}(X) & =H_{J}^{(2,0),(0,2)}(X)_{\mathbb{R}}, \\
H_{J}^{+}(X) & =H_{J}^{(1,1)}(X)_{\mathbb{R}}, \\
H_{-}^{J}(X) & =H_{(2,0),(0,2)}^{J}(X)_{\mathbb{R}}, \\
H_{+}^{J}(X) & =H_{(1,1)}^{J}(X)_{\mathbb{R}} .
\end{aligned}
$$

Remark 1.4. Let $S U(2) \times \mathbb{T}^{3}$ be the product of the special unitary group with the torus $\mathbb{T}^{3}=\mathbb{R}^{3} / \mathbb{Z}^{3}$. Let $\left\{e^{1}, \ldots, e^{6}\right\}$ be the global parallelism on $S U(2) \times \mathbb{T}^{3}$ satisfying the Maurer-Cartan equations

$$
\left\{\begin{array}{l}
d e^{1}=e^{2} \wedge e^{3}, \\
d e^{2}=-e^{1} \wedge e^{3}, \\
d e^{3}=e^{1} \wedge e^{2} \\
d e^{4}=d e^{5}=d e^{6}=0 .
\end{array}\right.
$$

Define the almost complex structure $J$ on $S U(2) \times \mathbb{T}^{3}$ by giving the following complex $(1,0)$-forms:

$$
\varphi^{1}=e^{1}+i e^{4}, \quad \varphi^{2}=e^{2}+i e^{5}, \quad \varphi^{3}=e^{3}+i e^{6}
$$

then (see [2, example 5.4]) we have that $J$ is a $\mathcal{C}^{\infty}$-full non- $\mathcal{C}^{\infty}$-pure almost complex structure. Hence, according to Proposition 1.3 $J$ is a pure non-full almost complex structure on $S U(2) \times \mathbb{T}^{3}$.

\section{COMPACT MANifolds}

2.1. J-Invariant cohomology classes. For general 2-cohomology classes on arbitrary manifolds, we can show

Proposition 2.1. Let $X$ be a $2 n$-dimensional manifold. Let $J$ be an almost complex structure on $X$ and $[\alpha] \in H^{2}(X ; \mathbb{R})$ such that $[\alpha]^{n} \neq 0$. Then

i) $[\alpha] \notin H_{J}^{-}(X)_{\mathbb{R}} \cap H_{J}^{+}(X)_{\mathbb{R}}$.

ii) Moreover, if $n$ is odd, then $[\alpha] \notin H_{J}^{-}(X)$. 
Proof. i) By contradiction. Let $[\alpha] \in H_{J}^{-}(X) \cap H_{J}^{+}(X)$; then $[\alpha]=\left[\alpha_{1}\right]=\left[\alpha_{2}\right]$, where $\alpha_{1}$ is $J$-anti-invariant and $\alpha_{2}$ is $J$-invariant. Then $\alpha_{1} \wedge \alpha_{2}^{n-1}$ is a $J$-antiinvariant $2 n$-form which does not vanish identically. Therefore, $J\left(\alpha_{1} \wedge \alpha_{2}^{n-1}\right)=$ $-\alpha_{1} \wedge \alpha_{2}^{n-1}$. Take $\left\{e_{1}, \ldots, e_{n}, J e_{1}, \ldots, J e_{n}\right\}$ a basis of $T_{p} X$. Then

$$
\begin{aligned}
J\left(\alpha_{1} \wedge \alpha_{2}^{n-1}\right)\left(e_{1}, \ldots, e_{n}, J e_{1}, \ldots, J e_{n}\right) & =\alpha_{1} \wedge \alpha_{2}^{n-1}\left(J e_{1}, \ldots, J e_{n},-e_{1}, \ldots,-e_{n}\right) \\
& =\alpha_{1} \wedge \alpha_{2}^{n-1}\left(e_{1}, \ldots, e_{n}, J e_{1}, \ldots, J e_{n}\right),
\end{aligned}
$$

i.e., $J\left(\alpha_{1} \wedge \alpha_{2}^{n-1}\right)=\alpha_{1} \wedge \alpha_{2}^{n-1}$. This is absurd.

ii) We observe that $\alpha^{n} \neq 0$ at some point (otherwise, $[\alpha]^{n}=0$ ). Assume by contradiction that $[\alpha] \in H_{J}^{-}(X)$. Then there exists $\varphi \in \Lambda_{J}^{-}(X)$ such that $[\alpha]=[\varphi]$; furthermore, there exists a point $p \in X$ such that $\varphi^{n}(p) \neq 0$. Since $\varphi \in \Lambda_{J}^{-}(X)$ and $n$ is odd, we have that $J \varphi^{n}=-\varphi^{n}$. Arguing as in the proof of i), we obtain that $\alpha^{n}=0$. This is absurd.

As a direct consequence of the last proposition, in the compact case we have the following.

Corollary 2.2. Let $(X, \omega)$ be a $2 n$-dimensional compact symplectic manifold, with $n$ odd. Let $J$ be an almost complex structure on $M$. Then $[\omega] \notin H_{J}^{-}(X)$.

The following provides an example of a 2-cohomology class $\mathfrak{a}$ on a 6 -dimensional compact nilmanifold $N$ endowed with a left-invariant almost complex structure $J$, which belongs to $H_{J}^{+}(N) \cap H_{J}^{-}(N)$ and whose square is non-zero; nevertheless, $\mathfrak{a}^{3}=0$, according to Proposition 2.1 .

2.2. Example. Let $\mathfrak{g}$ be the 6 -dimensional nilpotent Lie algebra whose dual vector space admits a basis $\left\{e^{1}, \ldots, e^{6}\right\}$ satisfying the following Maurer-Cartan equations:

$$
d e^{1}=0, \quad d e^{2}=0, \quad d e^{3}=e^{12}, \quad d e^{4}=e^{13}, \quad d e^{5}=e^{14}, \quad d e^{6}=e^{23},
$$

where $e^{i j}=e^{i} \wedge e^{j}$, and so on. Let $G$ be the connected and simply-connected Lie group having $\mathfrak{g}$ as a Lie algebra. Then, $G$ admits a uniform discrete subgroup $\Gamma$ and $N=\Gamma \backslash G$ is a 6 -dimensional nilmanifold. Let $J$ be the almost complex structure on $N$ defined by the following global $(1,0)$-forms:

$$
\psi^{1}=e^{1}+i e^{2}, \quad \psi^{2}=e^{4}+i e^{6}, \quad \psi^{3}=e^{3}+i e^{5} .
$$

By the Nomizu Theorem, it follows that $H^{\bullet}(\mathfrak{g}) \simeq H^{\bullet}(N ; \mathbb{R})$ and, consequently, $b_{1}(N)=2, b_{2}(N)=4$ and the $b_{3}(N)=6$. By a straightforward computation it follows that $0 \neq\left[e^{26}\right] \in H^{2}(N ; \mathbb{R})$ and $0 \neq\left[e^{15}\right] \in H^{2}(N ; \mathbb{R})$. Furthermore,

$$
\left[e^{26}\right]=\left[e^{26}-e^{14}\right]=\left[-\Re \mathfrak{e}\left(\psi^{1} \wedge \psi^{2}\right)\right], \quad\left[e^{26}\right]=\left[e^{26}+e^{14}\right]=\left[\Re \mathfrak{e}\left(\psi^{1} \wedge \bar{\psi}^{2}\right)\right]
$$

and

$$
\left[e^{15}\right]=\left[e^{15}+e^{23}\right]=\left[\Im \mathfrak{m}\left(\psi^{1} \wedge \psi^{3}\right)\right], \quad\left[e^{15}\right]=\left[e^{15}-e^{23}\right]=\left[\Im \mathfrak{m}\left(\bar{\psi}^{1} \wedge \psi^{3}\right)\right] .
$$

Therefore, $0 \neq\left[e^{26}\right] \in H_{J}^{+}(N) \cap H_{J}^{-}(N)$ and $0 \neq\left[e^{15}\right] \in H_{J}^{+}(N) \cap H_{J}^{-}(N)$. Set $\mathfrak{a}=\left[e^{26}-e^{15}\right] \in H_{J}^{+}(N) \cap H_{J}^{-}(N)$ and $\mathfrak{a}^{2}=2\left[e^{1256}\right] \neq 0$. 


\section{NON-COMPACT MANIFOLDS}

In this section we consider open manifolds (without boundary), namely manifolds without compact connected components. Starting with a symplectic 4-manifold, we have

Theorem 3.1. Let $(X, \omega)$ be a 4-dimensional open symplectic manifold. Then there exists an almost complex structure $J$ on $X$ such that $\omega \in \mathcal{Z}_{J}^{-}$, that is, $\omega$ is $J$-anti-invariant, if and only if the tangent bundle $T X$ is symplectically trivial.

Proof. $(\Leftarrow)$ Assume that $\left\{V_{1}, V_{2}, W_{1}, W_{2}\right\}$ is a global symplectic frame on $X$, namely

$$
\omega\left(V_{i}, W_{j}\right)=\delta_{i j}, \quad \omega\left(V_{i}, V_{j}\right)=\omega\left(W_{i}, W_{j}\right)=0, \quad i, j=1, \ldots, 4 .
$$

Define

$$
J V_{1}=V_{2}, \quad J W_{1}=-W_{2} .
$$

Then $\omega=V^{1} \wedge W^{1}+V^{2} \wedge W^{2}$, where $\left\{V^{1}, V^{2}, W^{1}, W^{2}\right\}$ is the dual frame of $\left\{V_{1}, V_{2}, W_{1}, W_{2}\right\}$, and it is immediate to check that $J \omega=-\omega$.

$(\Rightarrow)$ Assume that there exists an almost complex structure $J$ such that $J \omega=-\omega$. As $X$ is open we can choose a never vanishing vector field $V_{1}$ on $X$ and let $K$ be an $\omega$-calibrated almost complex structure on $X$. Since $\omega$ is $J$-anti-invariant, we have that

$$
\omega\left(V_{1}, J V_{1}\right)=0, \quad \omega\left(K V_{1}, J K V_{1}\right)=0 ;
$$

furthermore, $\omega\left(V_{1}, K V_{1}\right)>0$, with $K$ being $\omega$-calibrated, and, consequently, $\omega\left(J V_{1}, J K V_{1}\right)<0$. Then, by using the standard symplectic Gram-Schmidt process, we can extract a global symplectic basis from $\left\{V_{1}, J V_{1}, K V_{1}, J K V_{1}\right\}$.

Remark 3.2. We observe that the proof of $(\Leftarrow)$ holds for every $2 n$-dimensional symplectic manifold with a symplectically trivial tangent bundle, provided that $n$ is even. However a corollary of the proof of Proposition 2.1 says that symplectic forms on $2 n$-dimensional manifolds with $n$ odd are never $J$-anti-invariant.

The next result shows that the $(\Rightarrow)$ direction of Theorem 3.1 holds only in the 4-dimensional case. Namely,

Proposition 3.3. Let $\left(X^{2 n}, J\right)$ be an almost complex manifold such that the Pontrjagin class $p_{1}(T X) \neq 0$ and it admits a $J$-anti-invariant symplectic form $\alpha$. Then the open almost complex $(2 n+4 k)$-dimensional manifold $X \times \mathbb{C}^{2 k}$ has a symplectic anti-invariant form but $T\left(X \times \mathbb{C}^{4 k}\right)$ is non-trivial.

Proof. By assumption, there exists a symplectic form $\alpha \in \mathcal{Z}_{J}^{-}$. Let $\left(z_{1}, \ldots, z_{k}\right.$, $\left.w_{1}, \ldots, w_{k}\right)$ be coordinates on $\mathbb{C}^{2 k}$ and set $\omega=\sum_{j=1}^{k} d z_{j} \wedge d w_{j}$. Then $\alpha+\omega \in$ $\mathcal{Z}_{\hat{J}}^{-}\left(X \times \mathbb{C}^{2 k}\right)$, where $\hat{J}$ is the product almost complex structure on $X \times \mathbb{C}^{2 k}$.

We have $p_{1}\left(\left.T\left(X \times \mathbb{C}^{2 k}\right)\right|_{X \times\{0\}}\right) \in H^{4}(X)$ and $p_{1}\left(T X \oplus \mathbb{C}^{2 k}\right)=p_{1}(T X) \neq 0$ by assumption, and so the tangent bundle is non-trivial.

Example 3.4. As an application of Proposition 3.3, we take the Fermat quartic $X=\left\{\left[z_{0}: z_{1}: z_{2}: z_{3}\right] \in \mathbb{P}^{3}(\mathbb{C}) \mid z_{0}^{4}+z_{1}^{4}+z_{2}^{4}+z_{3}^{4}=0\right\}$. It turns out that the signature $\tau(X)=-16$ (see e.g. [14, p. 156]). Then, according to the Hirzebruch signature theorem, $p_{1}(T X)=3 \tau(X)=-48$. Furthermore, it is well known that $X$ has a nowhere vanishing holomorphic $(2,0)$-form $\Omega$. Hence, setting $\alpha=\frac{1}{2}(\Omega+\bar{\Omega})$, 
we have that $\alpha$ is a $J$-anti-invariant symplectic form on $X$. Therefore, $\alpha+\omega \in$ $\mathcal{Z}_{\hat{J}}^{-}\left(X \times \mathbb{C}^{2 k}\right)$, where $\hat{J}$ is the product almost complex structure on $X \times \mathbb{C}^{2 k}$ and $T\left(X \times \mathbb{C}^{2 k}\right)$ is non-trivial.

Theorem 3.5. Let $X$ be an open $2 n$-dimensional manifold with trivial tangent bundle and $n$ even. Then for any cohomology class $\mathfrak{a} \in H^{2}(X)$ there exists an almost complex structure $J$ and an anti-invariant symplectic form $\omega$ on $X$ such that $\mathfrak{a}=[\omega]$.

Proof. Let $\left\{V_{1}, \ldots, V_{n}, W_{1}, \ldots, W_{n}\right\}$ be a global frame on $X, J_{0}$ be the almost complex structure on $X$ defined as $J_{0} V_{i}=W_{i}, i=1, \ldots, n$, and $g$ be the Hermitian metric defined by requiring that $\left\{V_{1}, \ldots, V_{n}, W_{1}, \ldots, W_{n}\right\}$ is an orthonormal frame. Then the fundamental form $\omega_{0}$ of $g$ is an almost symplectic form with symplectically trivial tangent bundle. Denote by

$$
\begin{aligned}
& \mathbb{S}_{\text {symp }}^{\mathfrak{a}}=\{\text { symplectic forms } \omega \text { on } X \text { such that } \mathfrak{a}=[\omega]\}, \\
& \mathbb{S}_{\text {symp }}=\{\text { symplectic forms on } X\}, \\
& \mathcal{S}_{\text {symp }}=\{\text { almost symplectic forms on } X\} .
\end{aligned}
$$

Then $\mathbb{S}_{\text {symp }}^{\mathfrak{a}} \subset \mathbb{S}_{\text {symp }} \subset \mathcal{S}_{\text {symp }}$, and according to the $h$-principle (see [13] or [1], 10.2.2]) the inclusion $\mathbb{S}_{\text {symp }}^{\mathfrak{a}} \hookrightarrow \mathcal{S}_{\text {symp }}$ is a homotopy equivalence. In other words there exists a family $\omega_{t}$ of almost symplectic forms on $X$ with $\omega_{1}$ symplectic and $\mathfrak{a}=\left[\omega_{1}\right]$.

Denote by $E \rightarrow X \times[0,1]$ the bundle over $X \times[0,1]$ whose fibre is given by

$$
E_{x}=\left\{\text { ordered symplectic basis of } T_{x} X \text { with respect to } \omega_{t}\right\} .
$$

Since $\left(X, \omega_{0}\right)$ is symplectically trivial, then there exists a section of $E$ over $X \times\{0\}$. By the homotopy lifting property there is a section of $E$ over $X \times[0,1]$. In particular $\left(X, \omega_{1}\right)$ is symplectically trivial. Hence, in view of Remark 3.2 , it follows that there exists an almost complex structure $J$ such that $\omega_{1}$ is a $J$-anti-invariant form.

Concerning invariant cohomology classes, we have

Proposition 3.6. Let $(X, J)$ be an open almost complex manifold. Then given any non-zero class $\mathfrak{a} \in H^{2}(X ; \mathbb{R})$, there exists an almost complex structure $\hat{J}$ on $X$ which is homotopic to $J$ and such that $\mathfrak{a} \in H_{\hat{J}}^{+}(X)$ and $\mathfrak{a} \notin H_{\hat{J}}^{-}(X)$.

Proof. Let $0 \neq \mathfrak{a} \in H^{2}(X ; \mathbb{R})$; then by the $h$-principle as above (see the remark in [11] following Theorem 10.2.2), there exists a symplectic form $\omega$ and a homotopy equivalence of almost complex structures $\left\{\mathcal{J}_{t}\right\}$ for $t \in[0,1]$ such that

- $J \in\left\{\mathcal{J}_{t}\right\}$;

- $\mathfrak{a}=[\omega]$ and $\mathcal{J}_{1}$ is compatible with $\omega$.

Hence, $\mathfrak{a} \in H_{\mathcal{J}_{1}}^{+}(X)$. We claim that $\mathfrak{a} \notin H_{\mathcal{J}_{1}}^{-}(X)$. On the contrary, $\mathfrak{a} \in H_{\mathcal{J}_{1}}^{+}(X) \cap$ $H_{\mathcal{J}_{1}}^{-}(X)$; then $\mathfrak{a}=[\omega]=\left[\alpha_{-}\right]$, for suitable, $\alpha_{-} \in \mathcal{Z}^{-}$. Hence, we have

$$
J\left(\alpha_{-} \wedge \omega^{n-1}\right)=-\alpha_{-} \wedge \omega^{n-1}
$$

and thus the volume form

$$
\alpha_{-} \wedge \omega^{n-1}=0 .
$$

Therefore, as $\omega$ is non-degenerate, $\alpha_{-}$vanishes identically, and, consequently, $\mathfrak{a}=0$. This is absurd. 


\section{Analytic COntinuation of anti-InVARiant FORMS}

In this section we prove an analytic continuation result for anti-invariant closed forms on almost complex manifolds.

Theorem 4.1. Let $(X, J)$ be a connected 2 -dimensional almost complex manifold. Let $\alpha$ be a closed $J$-anti-invariant form on $X$ such that $\alpha=0$ on an open set $\mathcal{U} \subset X$. Then $\alpha=0$ on $X$.

The proof in the 4-dimensional case is a direct consequence of Hodge theory.

Proof (4-dimensional case). We may assume that $X=\mathbb{R}^{4}$. Take a $J$-Hermitian metric $g$ on $\mathbb{R}^{4}$; let $*_{g}$ be the Hodge operator and $\omega(\cdot, \cdot)=g(\cdot, J \cdot)$ be the fundamental form of $g$. Denote as usual by $\Lambda_{g}^{+}$the space of self-dual forms on $\left(\mathbb{R}^{4}, g\right)$. Then,

$$
\Lambda_{g}^{+}=\mathbb{R} \omega \oplus \Lambda_{J}^{-} .
$$

Hence, if $\alpha \in \mathcal{Z}^{-}$, then $d * \alpha=d \alpha=0$. Therefore $\Delta \alpha=0$, and by assumption $\alpha=0$ on $\mathcal{U}$. In view of the Unique Continuation Theorem by Aronszajn (see 4] and [15] or [6]) we obtain that $\alpha=0$ on $\mathbb{R}^{4}$.

The general case is more subtle but eventually reduces again to Aronszajn's Theorem.

Proof (general case). Let $\mathbb{D}$ be a $J$-holomorphic disk in $X$. Then following [17, Lemma 2.4.2, we can find a sufficiently small neighborhood $U$ of $\mathbb{D}$ and a smooth chart $\phi: U \rightarrow \mathbb{D} \times \mathbb{C}^{n-1} \subset \mathbb{C}^{n}$ such that $\phi(z)=(z, 0, \ldots, 0)$ and $d \phi(z)$ is holomorphic for all $z \in \mathbb{D}$. The second condition means that the pushed forward almost-complex structure $\phi_{*} J$ agrees with the standard product structure $i$ on $\mathbb{C}^{n}$ along $\mathbb{D} \times\{0\} \subset \mathbb{D} \times \mathbb{C}^{n-1}$. Hence, by working in this chart we can smoothly identify a small neighborhood of $\mathbb{D} \subset X$ with the total space of the complex vector bundle $\mathbb{D} \times \mathbb{R}^{2(n-1)} \equiv \mathbb{D} \times \mathbb{C}^{n-1} \rightarrow \mathbb{D}$ such that under the identification, $\left.J\right|_{\mathbb{D} \times\{0\}}$ coincides with the standard product structure.

Let $t:(z, w) \mapsto(z, t w)$ denote the scaling in the fibers.

We linearize $J$ on $\mathbb{D} \times \mathbb{C}^{n-1}$ by replacing $J$ by

$$
J_{0}=\lim _{t \rightarrow 0}\left(\frac{1}{t}\right)^{*} \circ J \circ t_{*} .
$$

Then $J_{0}$ is an almost complex structure on $\mathbb{D} \times \mathbb{C}^{n-1}$ invariant under scaling and restricting to the standard complex structure on the fibres $\{x\} \times \mathbb{C}^{n-1}$.

We can also replace $\alpha$ by $\alpha_{0}$ defined by

$$
\alpha_{0}(U, V)=\lim _{t \rightarrow 0}\left(\frac{1}{t}\right) \alpha(t U, t V) .
$$

Claim. $\alpha_{0}$ is a closed, smooth $J_{0}$-anti-invariant form on $\mathbb{D} \times \mathbb{C}^{n-1}$. It vanishes on the fibers $\{x\} \times \mathbb{C}^{n-1}$.

To justify the claim we compute the almost complex structures and anti-invariant forms explicitly in local coordinates. 
Let $\left(x_{1}, \ldots, x_{2 n}\right)$ be standard local coordinates in a neighborhood of a point $z \in \mathbb{D} \times\{0\} \subset \mathbb{D} \times \mathbb{R}^{2(n-1)}$. Then the scaling is given by

$$
t\left(x_{1}, \ldots, x_{2 n}\right)=\left(x_{1}, x_{2}, t x_{3}, \ldots, t x_{2 n}\right) .
$$

Denote the coordinate vector fields by $e_{i}=\frac{\partial}{\partial x_{i}}$. Then $t_{*} e_{i}=e_{i}$ for $i=1,2$ and $t_{*} e_{i}=t e_{i}$ for $i>2$. We can write

$$
\alpha=\sum_{i<j} a_{i j} d x_{i} \wedge d x_{j}
$$

and

$$
J e_{i}=\sum_{j} b_{i j} e_{j},
$$

where $a_{i j}$ and $b_{i j}$ are functions of $\left(x_{1}, \ldots, x_{2 n}\right)$. Now, by our choice of coordinates we have that $b_{1 j}(z)=\delta_{2 j}, b_{2 j}(z)=-\delta_{1 j}$ and $b_{i 1}(z)=b_{i 2}(z)=0$ for all $i>2$ at all points $z=\left(x_{1}, x_{2}, 0, \ldots, 0\right)$. Indeed, the matrix $b_{i j}(z)$ gives the standard complex structure at such points. Next, as anti-holomorphic forms necessarily vanish on complex planes, we have that $a_{12}(z)=0$.

We compute at a fixed point $x$ in the fiber over $z=\left(x_{1}, x_{2}\right) \in \mathbb{D}$ :

$$
\begin{gathered}
J_{t}\left(e_{1}(x)\right):=\left(\frac{1}{t}\right)^{*} J\left(t_{*} e_{1}(x)\right)=\left(\frac{1}{t}\right)^{*} J\left(e_{1}(t x)\right) \\
=\left(\frac{1}{t}\right)^{*} \sum_{j} b_{1 j}(t x) e_{j}=b_{11}(t x) e_{1}+b_{12}(t x) e_{2}+\sum_{j>2} b_{1 j}(t x) \frac{1}{t} e_{j} \\
\rightarrow e_{2}+\sum_{j, k>2} \frac{\partial b_{1 j}}{\partial x_{k}}(z) x_{k} e_{j}
\end{gathered}
$$

as $t \rightarrow 0$.

Similarly $J_{t}\left(e_{2}(x)\right):=\left(\frac{1}{t}\right)^{*} J\left(t_{*} e_{2}(x)\right)$ converges to

$$
-e_{1}+\sum_{j, k>2} \frac{\partial b_{2 j}}{\partial x_{k}}(z) x_{k} e_{j}
$$

as $t \rightarrow 0$.

For $i>2$ we have

$$
\begin{gathered}
J_{t}\left(e_{i}(x)\right):=\left(\frac{1}{t}\right)^{*} J\left(t_{*} e_{i}(x)\right)=\left(\frac{1}{t}\right)^{*} J(t x)\left(t e_{i}\right) \\
=\left(\frac{1}{t}\right)^{*} \sum_{j} b_{i j}(t x) t e_{j}=b_{i 1}(t x) t e_{1}+b_{i 2}(t x) t e_{2}+\sum_{j>2} b_{i j}(t x) e_{j} \\
\rightarrow \sum_{j>2} b_{i j}(z) e_{j}
\end{gathered}
$$

as $t \rightarrow 0$. Hence in these coordinates the fibers converge to complex vector spaces as $t \rightarrow 0$.

For the forms, we have

$$
\begin{aligned}
\alpha_{t}(x)\left(e_{1}, e_{2}\right) & :=\frac{1}{t} \alpha(t x)\left(e_{1}, e_{2}\right)=\frac{1}{t} a_{12}(t x) \\
& \rightarrow \sum_{k>2} \frac{\partial a_{12}}{\partial x_{k}}(z) x_{k}
\end{aligned}
$$

as $t \rightarrow 0$. 
For $i, j>2$ we have

$$
\alpha_{t}(x)\left(e_{i}, e_{j}\right):=\frac{1}{t} \alpha(t x)\left(t e_{i}, t e_{j}\right)=t a_{i j}(t x) \rightarrow 0
$$

as $t \rightarrow 0$.

Finally for $i>2$ we have

$$
\alpha_{t}(x)\left(e_{1}, e_{i}\right):=\frac{1}{t} \alpha(t x)\left(e_{1}, t e_{i}\right)=a_{1 i}(t x) \rightarrow a_{1 i}(z)
$$

as $t \rightarrow 0$ and

$$
\alpha_{t}(x)\left(e_{2}, e_{i}\right):=\frac{1}{t} \alpha(t x)\left(e_{2}, t e_{i}\right)=a_{2 i}(t x) \rightarrow a_{2 i}(z)
$$

as $t \rightarrow 0$. As the convergence here is smooth, $\alpha_{0}$ is closed and $J_{0}$-anti-invariant as claimed.

Now, by the computations above, to understand $\alpha_{0}$ we are only interested in the values of $a_{1 i}$ and $a_{2 i}$ for $i>2$ along the zero-section, and hence we will think of these only as functions of $x_{1}$ and $x_{2}$. The function $a_{12}$, on the other hand, we must still consider as a function of $x_{1}, \ldots, x_{2 n}$, although to simplify notation it is enough to consider the case when it is linear when restricted to the fibres. Similarly we may think of the partial derivatives $\frac{\partial b_{i j}}{\partial x_{k}}$ for $k>2$ as depending only on $\left(x_{1}, x_{2}\right)$.

There are relations amongst the functions above which we now detail. First of all, as a limit of closed forms, $\alpha_{0}$ is closed. Calculating its $d x_{1} \wedge d x_{2} \wedge d x_{i}$ component for $i>2$ we get

$$
\frac{\partial a_{12}}{\partial x_{i}}-\frac{\partial a_{1 i}}{\partial x_{2}}+\frac{\partial a_{2 i}}{\partial x_{1}}=0
$$

Now we consider the anti-invariance of $\alpha_{0}$ at points along the zero-section and obtain

$$
a_{1,(2 i-1)}=-a_{2,2 i}, \quad a_{1,2 i}=a_{2,(2 i-1)}
$$

for $i>1$. For instance, to prove the first of these, we use the formula $\alpha_{0}(z)\left(e_{1}, e_{2 i-1}\right)$ $=-\alpha_{0}(z)\left(e_{2}, e_{2 i}\right)$ for $z \in \mathbb{D}$.

Next, using our formulas for $\alpha_{0}$, we compute

$$
\begin{gathered}
0=\alpha_{0}\left(e_{1}, J_{0} e_{1}\right)=\alpha_{0}\left(e_{1}, e_{2}+\sum_{j, k>2} \frac{\partial b_{1 j}}{\partial x_{k}} x_{k} e_{j}\right) \\
=\sum_{k>2} \frac{\partial a_{12}}{\partial x_{k}} x_{k}+\sum_{j, k>2} a_{1 j} \frac{\partial b_{1 j}}{\partial x_{k}} x_{k} .
\end{gathered}
$$

As this holds for all choices of $x_{k}$ we obtain

$$
\frac{\partial a_{12}}{\partial x_{k}}=-\sum_{j>2} a_{1 j} \frac{\partial b_{1 j}}{\partial x_{k}}
$$

Applying equations (2), (12) and then (3) we obtain

$$
\frac{\partial a_{1,(2 i-1)}}{\partial x_{2}}-\frac{\partial a_{1,2 i}}{\partial x_{1}}=\frac{\partial a_{1,(2 i-1)}}{\partial x_{2}}-\frac{\partial a_{2,(2 i-1)}}{\partial x_{1}}=\frac{\partial a_{12}}{\partial x_{2 i-1}}=-\sum_{j>2} a_{1 j} \frac{\partial b_{1 j}}{\partial x_{2 i-1}}
$$

for all $i>1$, and similarly

$$
\frac{\partial a_{1,2 i}}{\partial x_{2}}+\frac{\partial a_{1,(2 i-1)}}{\partial x_{1}}=-\sum_{j>2} a_{1 j} \frac{\partial b_{1 j}}{\partial x_{2 i}}
$$


Differentiating (4) with respect to $x_{2}$ gives

$$
\frac{\partial^{2} a_{1,(2 i-1)}}{\partial x_{2}^{2}}-\frac{\partial^{2} a_{1,2 i}}{\partial x_{1} \partial x_{2}}=-\sum_{j>2}\left(\frac{\partial a_{1 j}}{\partial x_{2}} \frac{\partial b_{1 j}}{\partial x_{2 i-1}}+a_{1 j} \frac{\partial^{2} b_{1 j}}{\partial x_{2} \partial x_{2 i-1}}\right),
$$

and differentiating (5) with respect to $x_{1}$ gives

$$
\frac{\partial^{2} a_{1,2 i}}{\partial x_{1} \partial x_{2}}+\frac{\partial^{2} a_{1,(2 i-1)}}{\partial x_{1}^{2}}=-\sum_{j>2}\left(\frac{\partial a_{1 j}}{\partial x_{1}} \frac{\partial b_{1 j}}{\partial x_{2 i}}+a_{1 j} \frac{\partial^{2} b_{1 j}}{\partial x_{1} \partial x_{2 i}}\right) .
$$

Adding equations (6) and (7) gives a formula for $\Delta a_{1,(2 i-1)}$ in terms of the $a_{1 j}$ for $j>2$ and their first derivatives (recall that we think of $a_{1 j}$ and $a_{2 j}$ for $j>2$ as functions just of $\left.\left(x_{1}, x_{2}\right)\right)$. In fact, if we think of the $a_{1 j}, a_{2 k}$ as giving a function $a: \mathbb{D} \rightarrow \mathbb{R}^{4(n-1)},\left(x_{1}, x_{2}\right) \mapsto\left(a_{13}, \ldots, a_{1,2 n}, a_{23}, \ldots, a_{2,2 n}\right)$, then the above calculations give a bound on the Laplacian $\Delta a$ in terms of bounds on the function and its first derivatives $\frac{\partial a}{\partial x_{1}}$ and $\frac{\partial a}{\partial x_{2}}$. Thus by Aronszajn's Theorem the function $a$ satisfies a unique continuation theorem; in particular, if $a$ is identically zero near a point, then it is zero everywhere. Hence if the function $a$ vanishes near a point in $\mathbb{D}$, then it vanishes everywhere on $\mathbb{D}$. As $a_{12}$ is identically zero along the zero-section $\mathbb{D}$ (as anti-invariant forms vanish on holomorphic curves), we conclude that if $\alpha=0$ near a point of $\mathbb{D}$, then it is zero at all points of $\mathbb{D}$.

To conclude the proof we need the following lemma.

Lemma 4.2. Fix a Riemannian metric on $X$ defining a distance function $d$ and let $K \subset X$ be compact. There exists an $\epsilon>0$ such that for any $x, y \in K$ with $d(x, y)<\epsilon$ there exists a holomorphic disk $\mathbb{D}$ in $X$ passing through $x$ and $y$.

This follows as in [18, Theorem 3.1.1 (i).

The lemma, together with the unique continuation theorem established along disks, implies that the set of points where $\alpha$ vanishes identically in a neighborhood is closed. As it is clearly open we can conclude the proof of Theorem 4.1 .

\section{Closed FORMS OF RANK 0 AND 4}

In this section we construct closed forms which have everywhere rank 0 or 4 , have compact support near a submanifold $W^{2 n-2}$ of $X^{2 n}$, and are Poincaré dual to $[W] \in H_{2}(X)$. As explained in the introduction, although there are no pointwise obstructions, the unique continuation theorem, Theorem 4.1, shows that there do not exist corresponding anti-invariant almost complex structures. This is our main proposition.

Proposition 5.1. Let $W \subset X^{2 n}$ be a $(2 n-2)$-dimensional compact submanifold with trivial normal bundle $\nu(W)$ and $\sigma$ be a 1-form on $W$ such that $d \sigma$ never vanishes and $d(\sigma \wedge d \sigma)=0$. Then there is a compactly supported 2-form $\omega$ on the total space of $\nu(W)$ which everywhere has rank either 4 or 0 and is cohomologous to the Thom class $\tau$ of $\nu(W)$.

Proof. Let $\tau=r\left(x_{1}, y_{1}\right) d x_{1} \wedge d y_{1}$ be the local expression of the Thom class of $\nu(W)$, where $r\left(x_{1}, y_{1}\right)$ is a bump function such that $r=1$ on a neighborhood of $W$ in $T W$. Define

$$
\omega=\tau+d(r \sigma)
$$

Then, $\omega^{2}=\tau \wedge \tau+d r \wedge \sigma \wedge d r \wedge \sigma+r^{2} d \sigma \wedge d \sigma+2 \tau \wedge d r \wedge \sigma+2 r \tau \wedge d \sigma+2 r d r \wedge \sigma \wedge d \sigma$. 
Therefore,

$$
\omega^{2}=2 r(\tau+d r \wedge \sigma) \wedge d \sigma
$$

and, consequently,

$$
\omega^{2} \neq 0
$$

where $r \neq 0$. We have

$$
\begin{aligned}
\omega^{3}= & 2 r\left(\tau^{2} \wedge d \sigma+d r \wedge \sigma \wedge \tau \wedge d \sigma+\tau \wedge d \sigma \wedge d r \wedge \sigma+d r \wedge \sigma \wedge d \sigma \wedge d r \wedge \sigma\right) \\
& +2 r^{2}\left(\tau \wedge(d \sigma)^{2}+d r \wedge \sigma \wedge(d \sigma)^{2}\right)=0
\end{aligned}
$$

Therefore, at each point, $\omega$ has rank 4 or $\Omega=0$.

The condition in Proposition 5.1 that $W$ has a 1-form with certain properties is often satisfied. Indeed, let $\mathbb{T}^{6}=\mathbb{R}^{6} \backslash \mathbb{Z}^{6}$ be the 6 -dimensional standard torus. Denote by $\left(x_{1}, \ldots, x_{6}\right)$ the coordinates on $\mathbb{R}^{6}$. Let $W=\left\{\left[\left(x^{1}, \ldots, x^{6}\right)\right] \in \mathbb{T}^{6} \mid x_{5}=\right.$ $\left.x_{6}=0\right\}$. Then the Poincaré dual $\mathfrak{a}$ of $W$ is given by $d x_{5} \wedge d x_{6}$. Set

$$
\sigma=\cos \left(2 \pi x_{3}\right) d x_{1}+\sin \left(2 \pi x_{3}\right) d x_{2} .
$$

Then

$$
d \sigma=2 \pi\left(\sin \left(2 \pi x_{3}\right) d x_{1} \wedge d x_{3}-\cos \left(2 \pi x_{3}\right) d x_{2} \wedge d x_{3}\right)
$$

is never vanishing on $W$. We immediately obtain

$$
d(\sigma \wedge d \sigma)=0 \text {. }
$$

\section{ACKNOWLEDGEMENTS}

The authors would like to thank Fondazione Bruno Kessler-CIRM (Trento) for its support and very pleasant working environment. They would like to thank Daniele Angella for useful comments. They are also pleased to thank the referee for fruitful suggestions and remarks.

\section{REFERENCES}

[1] Daniele Angella and Adriano Tomassini, On cohomological decomposition of almost-complex manifolds and deformations, J. Symplectic Geom. 9 (2011), no. 3, 403-428. MR2817781 (2012k:32037)

[2] Daniele Angella and Adriano Tomassini, On the cohomology of almost-complex manifolds, Internat. J. Math. 23 (2012), no. 2, 1250019, 25, DOI 10.1142/S0129167X11007604. MR.2890470

[3] D. Angella, A. Tomassini, and W. Zhang, On cohomological decomposability of almost-Kähler structures, to appear in Proc. Amer. Math. Soc. DOI 10.1090/S0002-9939-2014-12049-1

[4] N. Aronszajn, A unique continuation theorem for solutions of elliptic partial differential equations or inequalities of second order, J. Math. Pures Appl. (9) 36 (1957), 235-249. MR0092067 (19,1056c)

[5] Raoul Bott and Loring W. Tu, Differential forms in algebraic topology, Graduate Texts in Mathematics, vol. 82, Springer-Verlag, New York-Berlin, 1982. MR658304 (83i:57016)

[6] Sylvain Cappell, Dennis DeTurck, Herman Gluck, and Edward Y. Miller, Cohomology of harmonic forms on Riemannian manifolds with boundary, Forum Math. 18 (2006), no. 6, 923-931, DOI 10.1515/FORUM.2006.046. MR2278607 (2008c:58003)

[7] J.-P. Demailly, Complex Analytic and Differential Geometry, http://www-fourier.ujfgrenoble.fr/ demailly/manuscripts/agbook.pdf, 2009.

[8] Georges de Rham, Differentiable manifolds, Forms, currents, harmonic forms; translated from the French by F. R. Smith, with an introduction by S. S. Chern. Grundlehren der Mathematischen Wissenschaften [Fundamental Principles of Mathematical Sciences], vol. 266, SpringerVerlag, Berlin, 1984. MR760450 (85m:58005)

[9] Tedi Draghici, Tian-Jun Li, and Weiyi Zhang, Symplectic forms and cohomology decomposition of almost complex four-manifolds, Int. Math. Res. Not. IMRN 1 (2010), 1-17, DOI 10.1093/imrn/rnp113. MR2576281 (2011g:53151) 
[10] T. Drăghici, T.-J. Li, and W. Zhang, On the J-anti-invariant cohomology of almost complex 4-manifolds, Q. J. Math. 64 (2013), no. 1, 83-111. MR3032090

[11] Y. Eliashberg and N. Mishachev, Introduction to the h-principle, Graduate Studies in Mathematics, vol. 48, American Mathematical Society, Providence, RI, 2002. MR.1909245 (2003g:53164)

[12] Anna Fino and Adriano Tomassini, On some cohomological properties of almost complex manifolds, J. Geom. Anal. 20 (2010), no. 1, 107-131, DOI 10.1007/s12220-009-9098-3. MR2574723(2010k:32036)

[13] M. L. Gromov, Stable mappings of foliations into manifolds (Russian), Izv. Akad. Nauk SSSR Ser. Mat. 33 (1969), 707-734. MR0263103 (41 \#7708)

[14] Dominic D. Joyce, Compact manifolds with special holonomy, Oxford Mathematical Monographs, Oxford University Press, Oxford, 2000. MR1787733 (2001k:53093)

[15] Jerry L. Kazdan, Unique continuation in geometry, Comm. Pure Appl. Math. 41 (1988), no. 5, 667-681, DOI 10.1002/cpa.3160410508. MR948075 (89k:35039)

[16] Tian-Jun Li and Weiyi Zhang, Comparing tamed and compatible symplectic cones and cohomological properties of almost complex manifolds, Comm. Anal. Geom. 17 (2009), no. 4, 651-683, DOI 10.4310/CAG.2009.v17.n4.a4. MR2601348(2011e:32036)

[17] Dusa McDuff and Dietmar Salamon, J-holomorphic curves and symplectic topology, American Mathematical Society Colloquium Publications, vol. 52, American Mathematical Society, Providence, RI, 2004. MR2045629(2004m:53154)

[18] Jean-Claude Sikorav, Some properties of holomorphic curves in almost complex manifolds, Holomorphic curves in symplectic geometry, Progr. Math., vol. 117, Birkhäuser, Basel, 1994, pp. 165-189. MR.1274929

Department of Mathematics, University of Notre Dame, Notre Dame, Indiana 46556

E-mail address: hind.1@nd.edu

Dipartimento di Matematica e Informatica, Università di Parma, Viale Parco Area Delle Scienze 53/A, 43124, Parma, Italy

E-mail address: costantino.medori@unipr.it

Dipartimento di Matematica e Informatica, Università di Parma, Viale Parco Area delle Scienze 53/A, 43124, Parma, Italy

E-mail address: adriano.tomassini@unipr.it 